\title{
InTouch Wearables: Aesthetic Design of Remote Touch in Child-Parent Relationships
}

\author{
Jinsil Hwaryoung Seo \\ Soft Interaction Lab, TAMU \\ College Station, USA \\ hwaryoung@tamu.edu
}

\author{
Annie Sungkajun \\ Soft Interaction Lab, TAMU \\ College Station, USA \\ flora.s_secret@tamu.edu
}

\author{
Meghan Cook \\ Soft Interaction Lab, TAMU \\ College Station, USA \\ mecook20@tamu.edu
}

\begin{abstract}
InTouch Wearables is a set of wearables that consist of dresses and shoulder pieces that allow mother and child to share remote touches through garments with ambient feedback. This was created to explore how remote touches can convey emotion and help people stay connected between remote locations. This project was created based on the lead artist's personal experience with her child. In InTouch Wearables, a parent can increase the vividness of her conversation with a child through contextualised touch, and the loved ones may enhance the affective tone of their communication using remote touch technology. All the electronic components of the garment for sensing human touches and actuating colour-changing garment are embedded on the main fabrics.
\end{abstract}

Remote touch. Child-parent relationship. Interactive wearables. Colour changing garment. Creative engagement.

\section{INTRODUCTION}

InTouch Wearables is a set of wearables that consist of dresses and wearable accessories that allow mother and child share remote touches through garments with ambient feedback. This includes a set of dresses and a set of shoulder pieces. This was developed to explore how remote touches can convey emotion and help people stay connected between remote locations. Human interpersonal touch plays an important role in our social interaction in expressing affect and fostering intimacy. It is a special conduit through which humans convey love, comfort, care, trust, support, and appreciation. Touch is immediate in that it carries emotional meaning in ways that words cannot express (Montagu 1986).

This project was created based on the lead artist's personal experience with her child. When her son was one-year-old, he stayed with his grandparents in Korea apart from the artist. As an artist, she wanted to capture that moment as wearable and tangible artworks exploring the concept of remote touch. The final outcomes and the process of InTouch Wearables helped the artist to get immersed in the remote parent-child relationship and overcome the emotional challenge.

The first set of InTouch Wearables consists of a mother's dress and a child's dress (Figure 1, Top). Only the child's dress has touch sensitive parts.

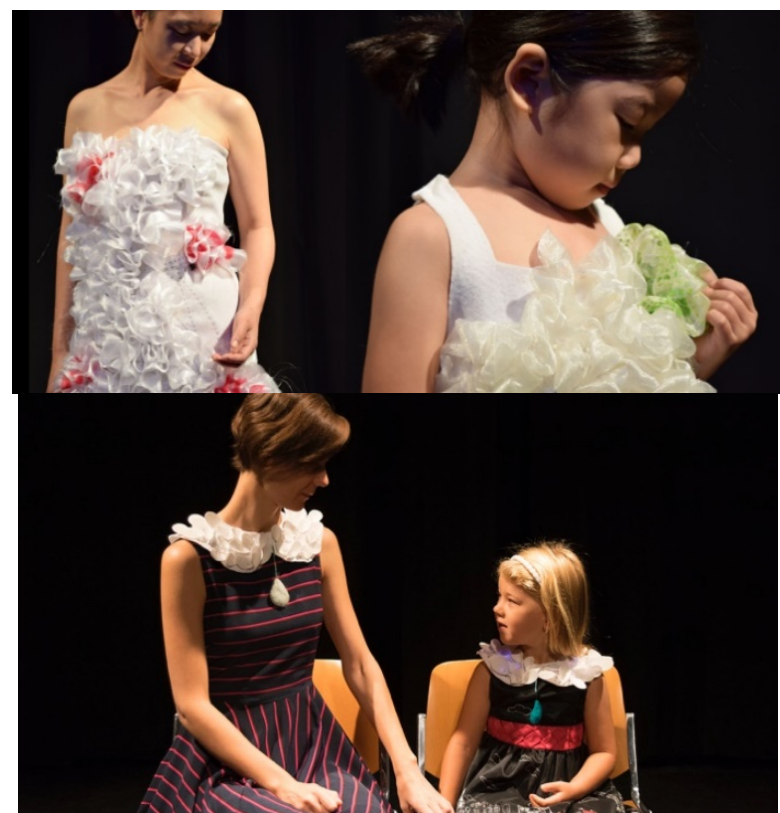

Figure 1: (Top) InTouch Wearables Dresses, (Bottom) InTouch Wearables Shoulder Pieces with a soft touch sensor

Whenever each touch area is being touched, a capacitive sensor embedded in the garment triggers degree of touch and the touch information is sent to the mapped area of the mother's dress and changes the colour of the area. The touch sensors on the child dress were distributed on the front side of the dress so that they detect non-intentional movements and contacts with others. The shoulder pieces of 
InTouch Wearables (Figure 1, Bottom) have soft touch sensors that are integrated into the design. We created a soft object that they can touch or caress when they want to send touch messages each other.

\section{IMPLEMENTATION}

\subsection{Soft Circuit}

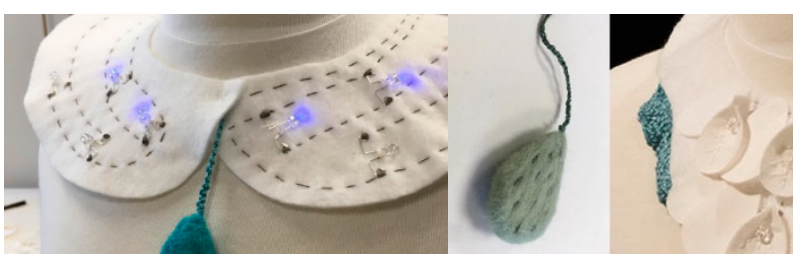

Figure 2: Soft Circuit of InTouch Wearables

The garments are constructed from iterative explorations of soft circuits using conductive fabric, yarn and thread as well as Lilypad Arduino with a capacitive sensing circuit and a heat control circuit.

\subsection{Networked Garments}

Within InTouch Wearables dresses, the mother's garment and the child garment communicate wirelessly via an Xbee and Lilypad Arduino. The child dress has five touch sensitive spots that look like flowers. When they are triggered by human touch, the green pattern of the flower changes its colour to yellow and the touch signal is sent to the mother's garment. The flower on the mother's dress mapped to the sensor on the child's dress changes its colour from dark pink to white. This creates not an alert rather an ambient experience. In InTouch Wearables shoulder pieces, the mother's and child's garments communicate via Bluetooth with Lilypad Arduino.

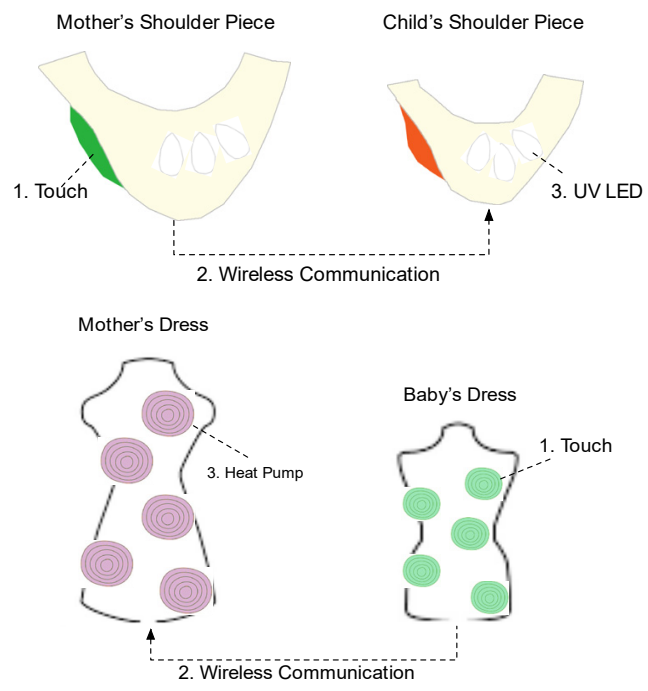

Figure 3: Network diagrams of dresses (Top) and shoulder piece (Bottom)

\subsection{Colour Changing Garments}

The colour changing aspect of the project has been utilised using thermochromic powders and invisible UV inks. Thermochromic powders are heat sensitive and the transition temperature to a different colour is $86 \mathrm{~F}$. For the child's dress, green colour powder was mixed with fabric medium and yellow acrylic paint. The mixed paint was applied to the fabric using a stencil technique. For the mother's dress, conductive thread was stitched on the surface of pink thermochromic paint and fabricated as a flower. The conductive thread was used as a heat source and connected to the heat control circuit. The colour change using thermal materials tends to be slow. It creates a passive and ambient wearable interface. This supports a mother feels always so close to the child's daily life including physical movements and interaction with surroundings. UV inks were applied on fabrics. The UV patterns appear when UV LEDs are on.

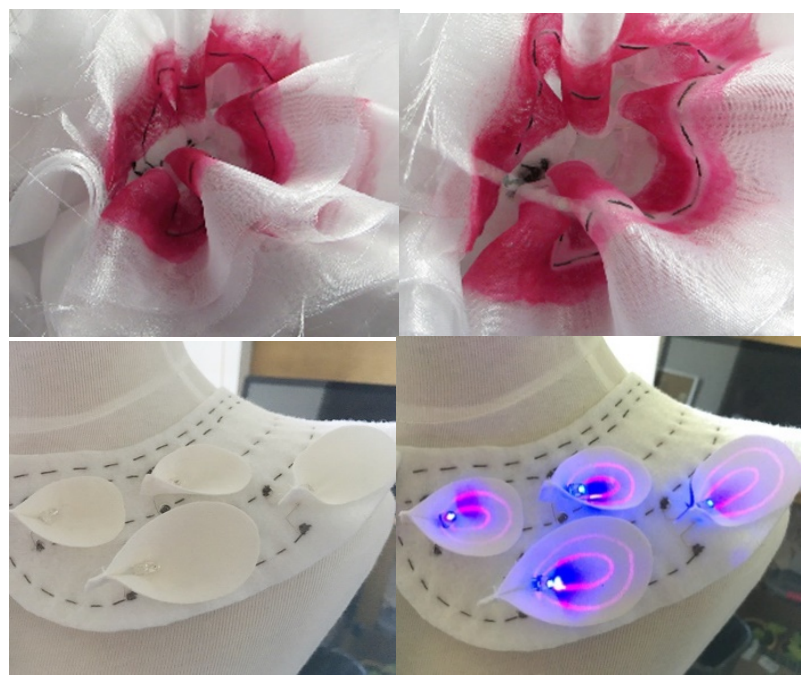

Figure 4: Colour changing garment using thermochromic powder (Top) and UV ink (Bottom)

\section{CONCLUSION}

InTouch Wearables explored the concept of remote touch in a parent and child relationship using colour changing garments. The artists took a poetic approach rather than a practical approach and the concept was realised as tangible/wearable forms and the technology was implemented to six wearable garments. This can be expanded to different designs (i.e., cushion or toy) and applications to investigate qualities of tactile experience.

\section{REFERENCES}

Montagu, A. (1986) Touching: The Human Significance of the Skin. Body, Mind \& Spirit. Harper Collins. 